\title{
Impacto de exportaciones primarias en el crecimiento económico del Ecuador: análisis econométrico desde Cobb Douglas, período 2000-2017
}

\section{Impact of primary exports on the economic growth of Ecuador: an econometric analysis from Cobb Douglas, 2000-2017}

María Andrea Alvarado Mora

Nandy Rossy Ullauri Martínez

Francisco Vladimir Benítez Luzuriaga

Universidad Técnica de Machala, Ecuador

Autor por correspondencia: maalvaradom_est@utmachala.edu.ec;

nullauri_est@utmachala.edu.ec; fbenitez@utmachala.edu.ec

Fecha de recepción: 10 de agosto del 2019 - Fecha de aceptación: 02 de octubre del 2019

\section{Resumen}

Las exportaciones de un país representan un sinnúmero de beneficios, tales como el ingreso de divisas, creación de fuentes de empleo, la reducción de costos, participación internacional, entre otros. Para el Ecuador las exportaciones primarias constituyen la base de la economía, por lo que creímos importante realizar un análisis del período 2000-2017 en función del modelo econométrico de Cobb Douglas. Las elasticidades de los tres productos principales de exportación del Ecuador señalan que el mayor aporte en expresiones elásticas en el período tomado para el análisis fue el banano (segundo rubro de mayor importancia en las exportaciones totales), el cual por cada punto porcentual generó aproximadamente un incremento de riquezas hacia el PIB de $0.16 \%$. Se pudo conocer, además, que al medir los rendimientos de escalas de los tres productos de exportación primaria tomadas en este artículo (crudo de petróleo, banano y camarón) para el crecimiento económico del Ecuador desde la perspectiva del PIB, este resultó de acuerdo a las propiedades de la función de producción de Cobb Douglas, en una economía de escalas decreciente, debido a la baja productividad e innovación, escenario que se repite en la mayoría de economías de américa latina, cuyo enfoque de exportaciones tiene como base a los commodities.

Palabras clave: exportaciones primarias; crecimiento económico; modelo econométrico de Cobb Douglas; commodities; elasticidad

\begin{abstract}
The exports of a country represent countless benefits, such as the entry of foreign exchange, creation of employment sources, cost reduction, international participation, among others. For Ecuador, primary exports are the basis of the economy, so we thought it important to carry out an analysis of the 2000-2017 period based on the Cobb Douglas econometric model. The elasticities of the three main export products of Ecuador indicate that the greatest contribution in elastic expressions in the period taken for the analysis was the banana (second most important item in
\end{abstract}


total exports), which generated approximately one percentage point for each percentage point. wealth increase towards GDP of $0.16 \%$. It was also known that when measuring the returns of scales of the three primary export products taken in this article (crude oil, bananas and shrimp) for the economic growth of Ecuador from the perspective of GDP, this resulted according to the properties of the Cobb Douglas production function, in a declining economy of scales, due to low productivity and innovation, a scenario that is repeated in most Latin American economies, whose export focus is based on commodities.

Key words: primary exports; economic growth; Cobb Douglas econometric model; commodities; elasticity

\section{Introducción}

Si una economía depende en gran medida de las exportaciones de las materias primas o de los productos parcialmente terminados, es mucho más probable que se vea afectada por variaciones en los precios mundiales y habría poco por hacer para evitar una disminución en la actividad comercial o incluso una crisis (Pacek, 2008).

Lograr crecimientos en los niveles de exportación para un país implica varios componentes que se deben analizar como ventajas comparativas, acceso a tecnologías, bloques comerciales, políticas administrativas locales, formas de financiación, ubicación geográfica, dotación de factores de producción o capital humano entre otros. En cada país estos elementos tienen un impacto de mayor o menor medida.

En la actualidad la ventaja competitiva, diversificación y oferta de bienes primarios, han tomado relevancia dentro del sector de las exportaciones como fuente generadora de divisas y por ende de desarrollo de las economías que dependen de esta actividad, que por lo general son países pertenecientes a América Latina; los cuales se enfrentan al reto de aumentar y adquirir mejores capacidades productivas, calidad y precio, que permita hacer más atractivos los bienes destinados a exportar.

La influencia de las exportaciones primarias hacia el crecimiento y desarrollo económico ha sido una temática de estudios desde hace aproximadamente un siglo, en donde se considera a todos los bienes abundantes de recursos naturales con ventajas productivas y calidad para ser exportados hacia el mercado internacional, y obtener en consecuencia crecimiento y diversificación económica (Caria, 2017).

Descendiendo a Ecuador, las exportaciones primarias en el 2017 significaron el $18 \%$ del PIB (Comercio Exterior, 2017), lo cual ratifica la repercusión que tienen para la economía nacional, dichas exportaciones se clasifican en petroleras y no petroleras; en la primera como su nombre lo dice son aquellas exportaciones del crudo de petróleo que se explota en el territorio ecuatoriano y representan el 6\%, y la segunda hace mención a todos los productos que no incluyan el crudo, que por lo general destaca el agro, en especial el banano y camarón las que después del petróleo generaron mejores beneficios al país.

Dicho esto, nace la importancia de la actual investigación, de conocer las participaciones en términos de elasticidades que tienen los tres principales bienes de las exportaciones primarias 
del Ecuador (crudo de petróleo, banano y camarón; medido en millones de dólares FOB), a través del uso metodológico econométrico de la función de producción de Cobb Douglas; la cual desde su creación hasta la actualidad ha tenido gran aceptación y uso para las estimaciones elásticas individuales (Ospina, 2017), en áreas de la microeconomía y macroeconomía (Vargas, 2014).

\section{Exportaciones y crecimiento económico}

De Gregorio (2012) define a las exportaciones como la demanda del resto del mundo hacia los bienes nacionales, que dependen de los precios que dichos bienes tengan y de los ingresos de los compradores internacionales, es decir, si el precio del bien a exportar baja, los países del mundo demandarán más de ellos, caso contrario pasa, si el precio de dicho bien aumenta. Además, las exportaciones, tienen un efecto multiplicador dentro de la economía, debido a que estas permiten a los países generar economías de escalas, innovación, tecnología, aumento de la ventaja comparativa e intensifica la producción manufacturera (Moreno, 2017).

Asimismo, en lo que refiere a exportaciones, si un país plantea y crea políticas favorables en donde se controle el exceso de importaciones y movimientos de capitales, este podrá gestar un mejor modelo de desarrollo económico, sumándose para ciertos contextos el mantener depreciado al tipo de cambio, que provoca que las exportaciones sean más atractivas y competitivas, constituyéndose a posteriori del intercambio de bienes y servicios, en divisas que el país exportador puede utilizar para el financiamiento de consumo e inversión (Morones, 2016).

Bayona (2016) menciona que una de las principales características para que exista crecimiento y desarrollo económico en los países exportadores, es que estos cuenten con recursos abundantes para explotar, ya sean petróleo, productos de explotación minera, productos agropecuarios y productos forestales. Sin embargo, pese a que Ecuador y el sector latinoamericano tienen gran dependencia exportable de este tipo de productos, según (Sach y Warner, 1997) mediante la modelación econométrica, demuestra que estos crecen en menor proporción que los países que exportan bienes manufacturados. Dicho postulado es apoyado por (Domínguez y Caria, 2016), quien diserta que los países que tienen alta dependía de la exportación de productos categorizados como primarios, con el tiempo presentan bajos cambios estructurales, los cuales implican la existencia de baja productividad, y baja generación de empleo.

En la misma línea, la baja productividad en los países dependientes de la exportación de productos primarios, se debe a que este está asociado a la variación que presentan los precios de los commodities, los cuales al estar altos, la tasa de crecimiento del país se mantiene alta, en cambio, cuando estos decaen, dicho crecimiento se ve contraído (Alvarado y Iglesias, 2017).

El comportamiento del crecimiento económico y el saldo de la balanza comercial es el resultado del dinamismo y movimiento que se le da a las exportaciones (Fraga y Moreno, 2015). Por tanto, para tener efectos positivos y mitigar el déficit y desbalances comerciales, es necesario que dentro de las políticas públicas se busque mejorar la oferta exportable, mediante cambios estructurales de la economía (Quiñones y Torres, 2015). 


\section{Exportaciones primarias}

Dentro del contexto de América Latina, existe una alta y significativa dependencia de estos países hacia las exportaciones de bienes primarios (Alvarado y Iglesias, 2017), en especial de productos como el petróleo, banano, café, camarón, cacao y flores, que junto a productos derivados de la manufactura agropecuaria tienen su prevalencia en la dinámica del comercio internacional y de la economía (Barrionuevo, Iborra, y Michelena, 2016).

No obstante, los países que tienen como base de su economía la producción y exportación de productos primarios, presentan a mediano y largo plazo problemas, que no solo van desde la falta de productividad e innovación tecnológica, sino que también tienen que lidiar con los efectos negativos ecológica y lo efectos negativos sociales de los procesos extractivos al momento de abastecer a las grandes economías como Estados Unidos y China (Samaniego, Vallejo, y Martínez, 2015; Vallejo, 2015).

En Ecuador, las exportaciones primarias junto con los productos manufacturados a base de recursos naturales, representan aproximadamente el $81 \%$ del sistema productivo agroexportador del país, lo que significa que dicho modelo es un rasgo característico del proceso de desarrollo económico ecuatoriano (Cypher y Alfaro, 2016), y los inconvenientes que se presenten al momento de producir y exportar, tendrá notables repercusiones en el crecimiento del producto interno bruto.

Los principales productos primarios que exporta el Ecuador según el BCE (2018) son el crudo de petróleo, banano y camarón, los cuales hasta el 2016 representan aproximadamente el $61 \%$ del total de las exportaciones que realizó el país (OEC, 2016). Dichos productos se exportan principalmente a Estados Unidos, Vietnam, Rusia, Colombia, Italia, España, Países Bajo, Alemania, China y Francia (Pro Ecuador, 2017). La figura 1 muestra el comportamiento de dichos bienes durante el período de estudios.

"El petróleo ha representado el principal rubro de exportación de Ecuador desde los años setenta" (Caria, 2017, pág. 125), convirtiéndose en la principal fuente de ingresos, y por tanto, el principal factor de dependencia económica que tiene el país por la alta entrada de divisas. El crudo exportable durante el período 2000-2015 tuvo un valor promedio de \$68 por barril, el cual fue superior a la década de los noventa con \$28 (Hurtado, 2016), permitiendo que la economía ecuatoriana tenga prosperidad; sin embargo a partir de diciembre del 2015 por las fluctuaciones de los commodities en el mercado internacional, dicho precio decayó a niveles muy bajos, postulándose en $\$ 33.67$, generando contracciones dentro de los ingresos que alimentan al producto interno bruto.

Por el lado del banano, es un bien exportable considerado como tradicional no petrolero (Caria, 2017), representando el 21\% de las exportaciones no petroleras ecuatorianas, convirtiéndose después del crudo de petróleo, en el segundo bien de mayor importancia para el dinamismo económico del Ecuador en términos de exportación, representando alrededor del 2\% del PIB, llevando al país a considerarse en la nación principal exportadora de banano en el 
mundo (Mosquera y Espinoza, 2016); por tanto, una disminución en su oferta exportable, significaría un fuerte golpe para los ingresos del país (Fierro y Villacres, 2014).

En cuanto al camarón, a mediados de la década de los 70’s, aparece como una nueva actividad económica y opción de exportación generadora de divisas, con características orientadas a la diversificación de los productos tradicionales exportables, mejorando a su vez la competitividad del país, convirtiéndolo en uno de los más destacados del mundo a través del tiempo (Guaipacha, Vélez, y Guime, 2017). En la actualidad este bien, ocupa el segundo puesto de las exportaciones no petroleras, conformado por alrededor de 39 empresas exportadoras y 1315 productores que poseen alrededor de 210000 hectáreas sembradas que generaron en el primer semestre del 2016 una cuantía de \$1116.31 millones FOB (Muñoz, Durán, y González, 2017); lo que indica y corrobora la importancia de dicho rubro para el dinamismo de la economía ecuatoriana.

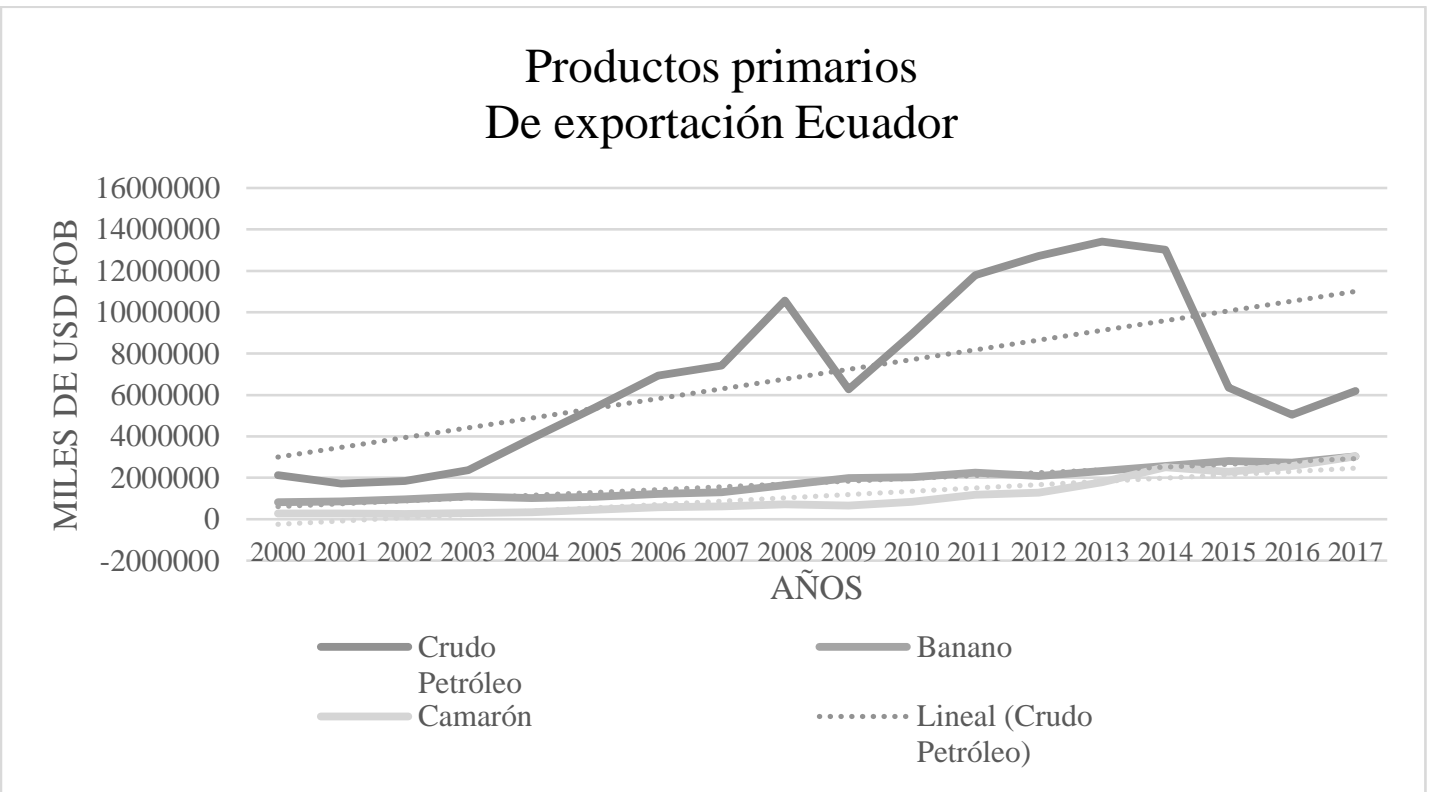

Ilustración \#1 El comportamiento de las variables crudo de petróleo, banano y camarón, durante el período de estudio denotaron tendencia positiva. Además se observa que la variable crudo de petróleo en dólares FOB tiene rubros más altos que las de banano y camarón, pese a que a partir del 2014 dichos valores decrecen debido a la caída del crudo de petróleo en el mercado internacional. Datos proporcionados por Banco Central del Ecuador.

\section{Metodología}

Dentro del campo de la econometría para la creación y adaptación de modelos econométricos es indispensable asegurarse la correcta especificación funcional, y para el caso de la presente temática en donde se requiere conocer el impacto que tienen las exportaciones primarias en la economía ecuatoriana (tomando en cuenta los bienes de mayor exportación del Ecuador: crudo de petróleo, banano y camarón) medido en términos de elasticidades, se debe usar la función de producción de Cobb Douglas (Montoya y Soto, 2011; Cedillo, Jumbo, y Campuzano, 2018), es decir, un modelo econométrico logarítmico. Por las características que presenta tendrá un enfoque cuantitativo con alcance descriptivo, con el que se demostrará según 
las propiedades que forman dicha función cuál de dichos bienes exportables ejercen mejores y mayores beneficios al crecimiento económico del Ecuador.

Las variables tomadas para formar el modelo son: PIB, exportaciones de crudo de petróleo, exportaciones de banano y exportaciones de camarón. Los datos fueron tomados del Banco Central del Ecuador (BCE, 2018), tomando como periodo de tiempo desde el 2000 hasta el 2017, y expresadas en miles de dólares americanos FOB.

\section{Y= f (exportación crudo de petróleo, exportación banano, exportación café)}

Respecto a la función de producción Cobb Douglas, esta es de naturaleza no lineal, debido a su estructura logarítmica (Cuevas, y otros, 2018), expresada:

$$
Y i=\beta 1 X_{2 i}^{\beta 2} X_{3 i}^{\beta 1} e^{u i}
$$

Si bien es cierto, la función de producción por poseer una estructura compleja y para que sea factible la aplicación de las propiedades de elasticidad de cada uno de los factores (X1, X2 y X3) se la debe modificar aplicando logaritmos, lo que permite que pase de un modelo convencional no lineal a un modelo doble log (Chiatchoua, Neme, y Valderrama, 2016), tal como se visualiza a continuación:

$$
\begin{gathered}
\ln Y i=\ln \beta_{1}+\beta_{2} \ln X_{2 i}+\beta_{\mathrm{n}} \ln X_{\mathrm{n} i}+u_{i} \\
=\beta_{0}+\beta_{2} \ln X_{2 i}+\beta_{3} \ln X_{3 i}+\beta_{4} \ln X_{4 i}+u_{i}
\end{gathered}
$$

En donde para este caso:

$\mathrm{Y}=\mathrm{PIB}$

$\mathrm{X} 2$ = Factor exportaciones crudo de petróleo

$\mathrm{X} 3=$ Factor exportaciones banano

X4= Factor exportaciones de camarón

$\mathrm{u}=$ Perturbación estocástica

$\mathrm{e}=$ Base logaritmo natural

Gujarati y Porter (2010) mencionan que la función de producción, también presenta beneficios que permiten observar los rendimientos de escalas de una economía, a través de la extracción y suma de coeficientes del modelo logarítmico, los cuales se lo presenciarán de acuerdo a las siguientes propiedades: si esta suma resulta 1, se refiere a rendimientos de escalas constantes; si es menor a 1, rendimientos de escalas decrecientes; y por último, si estos suman un valor superior a 1, se interpreta como una economía de rendimientos de escalas crecientes.

Adicionalmente, para que dicho modelado tenga validez estadística es necesario la aplicación de diferentes test que permitan una correcta estimación, entre las que se destaca, la prueba de Shapiro Wilk que mide la normalidad de las variables, la prueba de heterocedasticidad, colinealidad y significancia individual de cada variable; los cuales serán obtenidos a través del software estadístico Stata 14. 


\section{Resultados}

A priori los principales resultados que se obtuvieron del modelo logarítmico en el ajuste y explicación de las variables independientes hacia la variable dependiente, es que dichas regresoras explican en $\mathbf{9 8 . 8 1 \%}$ al crecimiento del PIB, es decir, que las variables sí generan un fuerte efecto dentro de la regresada.

Asimismo, y complementando al ajuste, el estadístico F de Fisher, demuestra que al ser mayor que 1 (387.33) el modelo en forma conjunta posee significancia y está en primera instancia bien especificado. Por el lado de la estimación de los parámetros, las variables logarítmicas de petróleo, banano y camarón, son estadísticamente significativas, debido a que estas tienen un p-valué menor al 5\%, lo que significa que los datos que recogen dichas variables acotan a que exista una estimación confiable.

Tabla. 1 Regresión múltiple logarítmica de lnPIB lnPetróleo lnBanano lnCamarón

\begin{tabular}{lcc}
\hline Variable & VIF & $\mathbf{1 / V I F}$ \\
\hline InBanano & 13.34 & 0.074939 \\
InCamarón & 12.66 & 0.078971 \\
InPetróleo & 2.11 & 0.473893 \\
Mean VIF & 9.37 & \\
\hline
\end{tabular}

Fuente: elaboración propia

Dentro del modelado econométrico, es necesario que se asegure una baja presencia de multicolinealidad, y para este caso en donde las variables en términos de logaritmos de la exportación del crudo de petróleo, banano y camarón que explican el crecimiento económico, evidencia un factor de inflación de la varianza (VIF = multicolinealidad) de 9.37, que al ser menor que 10, manifiesta que dicho problema no es preocupante (Salmerón, García, García, \& García, 2016). 
Tabla. 2

Prueba estadística de multicolinealidad VIF

\begin{tabular}{|c|c|c|c|c|c|c|}
\hline Source & SS & df & MS & & $\begin{array}{c}\text { Number of } \\
\text { obs }\end{array}$ & 18 \\
\hline & & & & & $\mathrm{F}(3,14)$ & 387.33 \\
\hline Model & $\begin{array}{c}.7905487 \\
95\end{array}$ & 3 & $\begin{array}{c}.2635162 \\
65\end{array}$ & & Prob $>F$ & 0.0000 \\
\hline \multirow[t]{2}{*}{ Residual } & $\begin{array}{c}.0095247 \\
24\end{array}$ & 14 & $\begin{array}{c}.0006803 \\
37\end{array}$ & & R-squared & 0.9881 \\
\hline & & & & & $\begin{array}{l}\text { Adj R- } \\
\text { squared }\end{array}$ & 0.9855 \\
\hline Total & $\begin{array}{c}.8000735 \\
19\end{array}$ & 17 & $\begin{array}{c}.0470631 \\
48\end{array}$ & & Root MSE & .02608 \\
\hline $\ln \mathrm{PIB}$ & Coef. & $\begin{array}{c}\text { Std.Err } \\
.\end{array}$ & t & $\begin{array}{l}\mathrm{P}>1 \mathrm{t} \\
1\end{array}$ & \multicolumn{2}{|c|}{ [95\% Conf. Interval] } \\
\hline lnPetroleo & .0548243 & $\begin{array}{c}.01347 \\
27\end{array}$ & 4.07 & 0.001 & .0259282 & $\begin{array}{c}.083720 \\
4\end{array}$ \\
\hline lnBanano & .157909 & $\begin{array}{c}.05204 \\
26\end{array}$ & 3.03 & 0.009 & .0462888 & $\begin{array}{c}.269529 \\
3\end{array}$ \\
\hline lnCamarón & .1395027 & $\begin{array}{c}.02642 \\
68\end{array}$ & 5.28 & 0.000 & .0828228 & $\begin{array}{c}196182 \\
6\end{array}$ \\
\hline _cons & 588.965 & $\begin{array}{c}.39314 \\
13\end{array}$ & 14.98 & $\begin{array}{l}0.00 \\
0\end{array}$ & 5.046 .446 & $\begin{array}{c}6.732 .8 \\
54\end{array}$ \\
\hline
\end{tabular}

Fuente: elaboración propia

Adicional a las pruebas calculadas, otro de los parámetros estadísticos que se determina para la fiabilidad en las estimaciones, es la normalidad de las variables, concepto que hace referencia a cómo se encuentran distribuidos los datos en cada uno de los factores tomados dentro de este modelo. Dicha normalidad parte de la hipótesis nula de que los datos tienen una distribución normal, por ende, para aceptar dicha condición el p-valué tiene que ponderarse por encima del 5\%, y para el caso de las variables seleccionadas del actual modelo, lnPIB, lnPetróleo, lnBanano y lnCamarón, poseen valores 14.27, 7.7\%, 10.12\% y $12.95 \%$ respectivamente, que son superiores al $5 \%$, lo que significa que aceptan hipótesis nula de normalidad.

Tabla. 3

Prueba de normalidad Shapiro Wilk

\begin{tabular}{lccccc}
\hline \multicolumn{1}{c}{ Variable } & Obs & $\mathbf{W}$ & $\mathbf{V}$ & $\mathbf{z}$ & Prob>z \\
\hline $\ln$ PIB & 18 & 0.92242 & 1.705 & 1.068 & 0.14267 \\
lnPetróleo & 18 & 0.90726 & 2.039 & 1.426 & 0.07699 \\
lnBanano & 18 & 0.91399 & 1.891 & 1.275 & 0.10117 \\
lnCamarón & 18 & 0.92005 & 1.757 & 1.129 & 0.12951 \\
\hline \multicolumn{5}{c}{ Fuente: elaboración propia }
\end{tabular}

Para determinar cuál de los distintos factores escogidos genera mayor impulso dentro del crecimiento económico del Ecuador, es necesario extraer cada uno de los coeficientes obtenidos de la regresión doble log y observar cuál de estos posee un coeficiente mayor, y para este caso, resulta que el factor de exportación primario que mayor incidencia tuvo para el crecimiento, es el 
banano (0.157909), el cual por cada punto porcentual que se incremente, acotó en aproximadamente $0.16 \%$ en el crecimiento.

Por la misma vía, el segundo factor de mayor incidencia hacia el crecimiento económico (para este caso) es la exportación de camarón, el cual presenta un coeficiente de 0.1395027 , significando que por cada $1 \%$ que se aumente dicho factor, el crecimiento económico experimentará alrededor de $0.14 \%$ de aumento. En cambio, el factor que menos incidencia presentó hacia el crecimiento económico ecuatoriano fue la exportación del crudo de petróleo; el cual al ejercerse un aumento del 1\%, solamente generó dentro de la economía un crecimiento de aproximadamente $0.055 \%$; resultado minúsculo en contraste con los otros dos factores considerados para el análisis.

Es pertinente mencionar, que pese a que en el gráfico 1 se muestra que en valores monetarios FOB el crudo de petróleo es el bien que mayor se exporta, un aumento de este en términos de elasticidades no genera una variación incidente para el crecimiento económico, lo cual se puede explicar ceteris paribus a los precios que este ha presentado durante el período tomado para el análisis, que en ocasiones ha resultado ser muy bajo y no ha presentado beneficios económicos para el país.

\section{$\ln (\mathrm{PIB})=5.88965$ + 0.0548243 $(\ln$ Petróleo $)$ + 0.157909 $(\operatorname{lnBanano})+0.1395027(\ln$ Camarón $)+\mathrm{u}$}

En cuanto al rendimiento de escalas, la suma de los coeficientes de lnPetróleo, lnBanano y lnCamarón, $(0.055+0.16+0.14)$, resulta aproximadamente 0.36 , lo que significa según las propiedades de la función de producción de Cobb Douglas, que las exportaciones de estos productos primarios seleccionados, resultan para el crecimiento una economía de escalas decrecientes.

\section{Conclusiones}

Teóricamente, las exportaciones son un factor incidente para la dinamización de la economía, y las variaciones que existan en esta variable generarán cambios significativos en el crecimiento económico, como también el desarrollo de tecnología, innovación, aumento de productividad y creación de empleos. Dentro de las exportaciones, se tiene el tipo de exportaciones primarias, de la cual depende el Ecuador y la mayor parte de los países de Latinoamérica, que si bien es cierto le significa un fuerte aporte para el producto interno bruto, pero al ser dependiente del precio de los commodities internacionales, al bajar dicho precio, los ingresos para el país decaerán significativamente.

Las elasticidades de los tres productos principales de exportación del Ecuador, dieron como resultados, que el mayor aporte en expresiones elásticas en el período tomado para el análisis fue el banano (segundo rubro de mayor importancia en las exportaciones totales), el cual por cada punto porcentual que este tuvo de aumento, generó aproximadamente un incremento de riquezas hacia el PIB de $0.16 \%$. Cabe señalar que a pesar de que el crudo de petróleo según datos obtenidos por el BCE, fue el que aportó mayores ingresos en dólares FOB para la economía ecuatoriana, en contraste al banano y camarón, este ejerció menor aporte por cada punto porcentual que se haya aumentado en las exportaciones por barril. Ceteris paribus, se presume 
que dicho fenómeno se da por la volatilidad de precios del crudo de petróleo sufrida en el mercado internacional.

Finalmente, al medir los rendimientos de escalas de los tres productos de exportación primaria tomadas en este artículo (crudo de petróleo, banano y camarón) para el crecimiento económico del Ecuador desde la perspectiva del PIB, este resultó de acuerdo a las propiedades de la función de producción de Cobb Douglas, en una economía de escalas decreciente. Resultado que no se presenta disímil a lo avistado en la teoría, en la que menciona que, si bien es cierto, las exportaciones primarias son una de las principales fuentes de ingreso de las economías latinas, a mediano y largo plazo presenta problemas en la oferta exportable por la baja productividad e innovación.

\section{Bibliografía}

Alvarado, R., \& Iglesias, S. (2017). Sector externo, restricciones y crecimiento económico en Ecuador. Problemas de desarrollo, 83-106. Obtenido de https://www.sciencedirect.com/science/article/pii/S030170361730041X

Barrionuevo, M., Iborra, M., \& Michelena, G. (2016). 24 oportunidades: un análisis del potencial exportador de las provincias argentinas. Revista Argentina de Economía Internacional, 20-34. Obtenido de http://www.cei.gob.ar/userfiles/RAEI\%205\%20$\% 20$ articulo\%202.pdf

Bayona, E. (2016). Producción de carbón y crecimiento económico en la región minera del Caribe colombiano. Revista de economía del caribe, 1-38. Obtenido de http://rcientificas.uninorte.edu.co/index.php/economia/article/viewFile/8452/pdf_270

BCE. (1 de diciembre de 2018). Banco Central del Ecuador. Obtenido de https://www.bce.fin.ec/index.php/component/search/?searchword=exportaciones\&orderin $\mathrm{g}=$ newest $\&$ searchphrase $=$ all $\&$ limit $=100$

BCE. (2018). Banco Central del Ecuador. Obtenido de https://www.bce.fin.ec/index.php/component/weblinks/weblink/21-informacioneconomica/294

Camino, S. (2017). Estimación de una función de producción y análisis de la productividad: el sector de innovación global en mercados locales. Estudios Gerenciales, 400-411. doi:https://doi.org/10.1016/j.estger.2017.10.004

Caria, S. (2017). El petróleo en Ecuador, 2000-2015: ¿maldición, bendición o simple recurso? Revista Iberoamericana de Estudios de Desarrollo, 124-147. Obtenido de https://www.researchgate.net/publication/320903694_El_petroleo_en_Ecuador_20002015_maldicion_bendicion_o_simple_recurso

Cedillo, L., Jumbo, K., \& Campuzano, J. (2018). Crecimiento económico del Ecuador: análisis econométrico desde Cobb Douglas, período 1990-2016. ESPACIOS, 6-12.

Chiatchoua, C., Neme, O., \& Valderrama, A. (2016). Inversión Extranjera Directa y empleo en México: análisis sectorial. Economía Informa, 40-59. doi:https://doi.org/10.1016/j.ecin.2016.04.004

Comercio Exterior. (2017). Ministerio de Comercio Exterior. Obtenido de https://www.comercioexterior.gob.ec/ecuador-aumenta-sus-exportaciones-al-mundo/

Cuevas, V., Loayza, A., Astengo, H., Moreno, T., Borja, M., Reyes, J., \& González, D. (2018). nálisis de la función de producción de leche en el sistema bovinos doble propósito en 
Ahome, Sinaloa. Revista mexicana de ciencias pecuarias, 376-386. doi:376http://dx.doi.org/10.22319/rmcp.v9i2.4545

Cypher, J., \& Alfaro, Y. (2016). Triángulo del neo-desarrollismo en Ecuador. Revista Problemas del Desarrollo, 161-184. Obtenido de http://www.scielo.org.mx/pdf/prode/v47n185/03017036-prode-47-185-00161.pdf

De Gregorio, J. (2012). Exportaciones. En J. De Gregorio, Macroeconomía, Teoría y Políticas (pág. 218). Santiago de Chile: José De Gregorio.

Domínguez, R., \& Caria, S. (2016). Ecuador en la trampa de la renta media. Revista Problemas del Desarrollo, 89-112. Obtenido de http://www.scielo.org.mx/pdf/prode/v47n187/03017036-prode-47-187-00089.pdf

Fierro, I., \& Villacres, C. (2014). Diagnóstico de la cadena logística de exportación del banano ecuatoriano hacia los Estados Unidos de América. Saber, ciencia y libertad, 77-89. Obtenido de https://revistas.unilibre.edu.co/index.php/saber/article/view/1985/1487

Fraga, C., \& Moreno, J. (2015). Exportaciones, términos de intercambio y ciclos de crecimiento económico de México y Brasil . EconoQuantum, 71-95. Obtenido de http://www.scielo.org.mx/pdf/ecoqu/v12n1/1870-6622-ecoqu-12-01-00071.pdf

Guaipacha, J., Vélez, C., \& Guime, J. (2017). Estudio de mercado internacional para la exportación del camarón ecuatoriano hacia el mercado español. Congreso Internacional en Administración de Negocios Internacionales, 378-391. Obtenido de https://dialnet.unirioja.es/servlet/articulo?codigo $=6290961$

Gujarati, D., \& Porter, D. (2010). La función de producción Cobb-Douglas: más sobre la forma funcional. En D. Gujarati, \& D. Porter, Econometría (págs. 207-208). México: McGrawHill.

Hurtado, F. (2016). Ecuador: innovación y emprendimiento para reinventar un modelo primario exportador. Santiago de Chile: Konrad-Adenauer-Stiftung e.V. Obtenido de http://www.bivica.org/upload/innovacion-emprendimiento.pdf\#page=136

Montoya, O., \& Soto, J. (2011). Estimación de la eficiencia técnica de las economías de los departamentos cafeteros de Colombia aplicando la función Cobb Douglas translogarítmica con fronteras estocásticas y datos de panel. Scientia Et Technica, 83-88. Obtenido de http://www.redalyc.org/articulo.oa?id=84921327015

Moreno, J. (2017). Inversión, cambio estructural y crecimiento. CEPAL, 1-37. Obtenido de https://www.researchgate.net/profile/Juan_Moreno-

Brid/publication/320414078_Inversion_cambio_estructural_y_crecimiento/links/59e4348 3a6fdcc7154dbbe78/Inversion-cambio-estructural-y-crecimiento.pdf

Morones, A. (2016). Crecimiento económico en México: restricción por la balanza de pagos. Ensayos. Revista de economía, 39-58\}. Obtenido de http://www.scielo.org.mx/scielo.php?pid=S2448$84022016000100039 \&$ script $=$ sci_arttext

Mosquera, M. A., \& Espinoza, J. (2016). Efectos de los TLC suscritos por Colombia con EE.UU. y UE en las exportaciones de banano ecuatoriano. Período 2001-2014. Valor Agregado, 73-166. Obtenido de http://www.valoragregado.ec/articulos/ValorAgregado06\%20\%20Art.\%203\%20Mosquera\%20y\%20Espinoza.pdf

Muñoz, M., Durán, F., \& Gónzalez, M. (2017). Análisis del sector camaronero ecuatoriano y sus ventajas competitivas y comparativas para encarar un mercado internacional competitivo. Conference Proceedings UTMACH, 1-8. Obtenido de 
http://investigacion.utmachala.edu.ec/proceedings/index.php/utmach/article/view/221/19 2

OEC. (2016). The Observatory of Economic Complexity. Obtenido de https://atlas.media.mit.edu/es/profile/country/ecu/\#Exportaciones

Ospina, J. (2017). The Cobb-Douglas function for a continuum model. Cuadernos de Economía, 1-18. Obtenido de http://www.redalyc.org/articulo.oa?id=282146950001

Pacek, N. y. (2008). Oportunidades en los mercados emergentes. Cuatro media.

Pro Ecuador. (2017). Boletín Mensual de Comercio Exterior. Dirección de Inteligencia Comercial e Inversiones, 1-20. Obtenido de http://portal.uasb.edu.ec/UserFiles/385/File/PRO\%20ECUADOR\%20SEPTOCT\%202017.pdf

Quiñones, N., \& Torres, R. (2015). Crecimiento y déficit comercial sostenible en Cuba: el papel de las exportaciones de bienes. Economía y Desarrollo, 141-158. Obtenido de http://scielo.sld.cu/pdf/eyd/v153s1/eyd09s15.pdf

Sach, J., \& Warner, A. (1997). Natural resource abundance and econonomic growth. Center for International Development and Harvard Institute for International Development, 1-36. Obtenido https://pdfs.semanticscholar.org/7b14/045909f42117197b82a910782ab68330a3e7.pdf

Salmerón, R., García, C., García, J., \& García, C. (2016). Treatment of collinearity through orthogonal regression: an economic application. Boletín de Estadística e Investigación Operativa, 184-202. Obtenido de http://www.seio.es/BBEIO/BEIOVol32Num3/files/assets/common/downloads/publicatio n.pdf\#page $=9$

Samaniego, P., Vallejo, M., \& Martínez, J. (2015). Desequilibrios en la balanza comercial andina: ¿se ajustan biofísicamente? Revista Iberoamericana de Economía Ecológica, 163185. Obtenido de https://dialnet.unirioja.es/servlet/articulo?codigo=5200403

Vallejo, M. C. (2015). Perfiles metabólicos de tres economías andinas: Colombia, Ecuador y Perú. Quito: FLACSO Ecuador. Obtenido de http://biblio.flacsoandes.edu.ec/catalog/resGet.php?resId=56208

Vargas, B. (2014). La Función de producción Cobb - Douglas. Fides et ratio, 67-74. Obtenido de http://www.revistasbolivianas.org.bo/pdf/rfer/v8n8/v8n8_a06.pdf 